\title{
Behavioral Effects of High-Strength Static Magnetic Fields on Rats
}

\author{
Thomas A. Houpt, ${ }^{1}$ David W. Pittman, ${ }^{2}$ Jan M. Barranco, ${ }^{1}$ Erin H. Brooks, ${ }^{2}$ and James C. Smith ${ }^{2}$ \\ Departments of ${ }^{1}$ Biological Science and ${ }^{2}$ Psychology, Program in Neuroscience, The Florida State University, Tallahassee, Florida 32306
}

\begin{abstract}
Advances in magnetic resonance imaging are driving the development of more powerful and higher-resolution machines with highstrength static magnetic fields. The behavioral effects of high-strength magnetic fields are largely uncharacterized, although restraint within a 9.4 T magnetic field is sufficient to induce a conditioned taste aversion (CTA) and induce brainstem expression of c-Fos in rats. To determine whether the behavioral effects of static magnetic fields are dependent on field strength, duration of exposure, and orientation with the field, rats were restrained within the bore of 7 or $14 \mathrm{~T}$ superconducting magnets for variable durations. Behavioral effects were assessed by scoring locomotor activity after release from the magnetic field and measuring CTA acquisition after pairing intake of a palatable glucose and saccharin $(\mathrm{G}+\mathrm{S})$ solution with magnetic field exposure. Magnetic field exposure at either 7 or $14 \mathrm{~T}$ suppressed rearing and induced tight circling. The direction of the circling was dependent on the rat's orientation within the magnetic field: if exposed head-up, rats circled counterclockwise; if exposed head-down, rats circled clockwise. CTA was induced after three pairings of taste and 30 min of $7 \mathrm{~T}$ exposure or after a single pairing of $\mathrm{G}+\mathrm{S}$ and $1 \mathrm{~min}$ of $14 \mathrm{~T}$ exposure. These results suggest that magnetic field exposure has graded effects on rat behavior. We hypothesize that restraint with high-strength magnetic fields causes vestibular stimulation resulting in locomotor circling and CTA acquisition.
\end{abstract}

Key words: conditioned taste aversion; vestibular; circling; rearing; magnetic resonance imaging; magnet

\section{Introduction}

Advances in magnetic resonance imaging (MRI) are driving the development of more powerful and higher-resolution MRI machines. Although MRI machines with static magnetic fields of 1-2 $\mathrm{T}$ and resolutions of $2 \mathrm{~mm}^{3}$ are standard in clinical use, higher resolution requires stronger magnetic fields of 4-9 T (Narasimhan and Jacobs, 1996).

The effects of high-strength static magnetic fields on mammals are largely unknown, and there is no evidence of long-term toxic effects of magnetic field-exposure in humans and rats. In humans, no acute aversive effects or sensations have been reported after exposure to magnetic fields of $\leq 1.5 \mathrm{~T}$ (Schenck et al., 1992; Winther et al., 1999). In rats, standard MRI protocols conducted from 0.15 to 1.89 T have been reported to have no effect on a variety of behavioral tasks (Innis et al., 1986; Ossenkopp et al., 1986; Messmer et al., 1987).

At higher field strengths, however, there have been reports of vertigo and nausea in workers around large magnets, for example, in a safety study of an early 4 T MRI machine (Schenck et al., 1992). Likewise, acute behavioral and neural effects on rats become apparent at higher field strengths (Weiss et al., 1992). Our laboratories have shown that a 30 min restraint of a rat within a 9.4 $\mathrm{T}$ superconducting magnet can induce circling locomotor activity (Snyder et al., 2000), conditioned taste aversion (CTA)

\footnotetext{
Received Jan. 19, 2001; revised 0ct. 22, 2002; accepted 0ct. 22, 2002.

This work was supported by National Institute on Deafness and Other Communication Disorders Grant 03198. We thank Drs. Timothy Cross, Rijang Fu, and Zhehong Gu of the United States National High Magnetic Field Laboratory for providing access to the magnets, Dr. Jeong Won Jahng for assistance and a reading of this manuscript, and Dr. Raymond Bye for support and encouragement.

Correspondence should be addressed to Thomas A. Houpt, Department of Biological Science, BRF 209 MC 4340, The Florida State University, Tallahassee, FL 32306-4340. E-mail: houpt@neuro.fsu.edu.

Copyright $\odot 2003$ Society for Neuroscience $\quad 0270-6474 / 03 / 231498-08 \$ 15.00 / 0$
}

(Nolte et al., 1998), and c-Fos expression (Snyder et al., 2000). CTA is a form of associative learning in which an animal learns to avoid the taste of a food previously paired with a toxic postingestive effect or nausea-inducing stimulus such as rotation. After intake of a highly palatable glucose plus saccharin solution $(G+S)$ was paired one to three times with restraint within the 9.4 $\mathrm{T}$ magnetic field, rats decreased their intake of $\mathrm{G}+\mathrm{S}$ relative to water for several days (Nolte et al., 1998). Because the magnetic field induced a CTA, it may have a visceral or vestibular effect that the rat can associate with the novel taste of $\mathrm{G}+\mathrm{S}$. Consistent with this hypothesis, $30 \mathrm{~min}$ of $9.4 \mathrm{~T}$ exposure induced significant c-Fos immunoreactivity in visceral and vestibular nuclei of the brainstem (Snyder et al., 2000).

Here we extend the previous behavioral results by using 7 and $14 \mathrm{~T}$ magnets to quantify the effects of different magnetic field strengths on circling behavior and CTA acquisition and to determine the thresholds of magnetic field intensity and duration of exposure sufficient to induce behavioral effects. The magnetic field exposure paired with the taste varied in four ways: field strength (7 vs $14 \mathrm{~T}$ ), number of pairings (one vs three), duration of exposure ( $0-30 \mathrm{~min}$ ), and orientation of the rat (head-up vs head-down within the magnetic field). To compare the results of the current study with our previously published data, we attempted to replicate as closely as possible the procedures used previously (Nolte et al., 1998) and included the data from the previous 9.4 T experiment in our analysis for comparison.

\section{Materials and Methods}

Subjects. Male Sprague Dawley rats (175-200 gm; Charles River Laboratories, Wilmington, MA) were housed individually in plastic cages in a temperature-controlled colony room at the National High Magnetic Field Laboratory at The Florida State University. The rats had a $12 \mathrm{hr}$ light/dark cycle with lights on at 8:00 A.M. All conditioning trials were 
conducted during the light cycle. The rats had access to pelleted Purina (St. Louis, MO) Rat Chow 5001 and deionized-distilled water ad libitum except where specified otherwise. Four days before the conditioning day, the rats were placed on a water-deprivation schedule under which they received daily water access in one drinking session. The initial session was $1 \mathrm{hr}$, and the session times were diminished each day so that the day before conditioning the rats received their water in a 10 min session.

Conditioning procedure. The procedure described previously (Nolte et al., 1998) was replicated in the present experiments. The conditioned stimulus (CS) was a solution of $30 \mathrm{gm}$ of glucose and $1.25 \mathrm{gm}$ of sodium saccharin mixed in 11 of deionized-distilled water $(G+S)$. The rats were allowed $10 \mathrm{~min}$ for access to the $\mathrm{G}+\mathrm{S}$. The unconditioned stimulus (US), which followed immediately after the CS, was a 30 min exposure in one of the two superconducting magnets. To expose the rats in the vertical bores of the magnets, rats were placed individually in a Plexiglas restraining tube that had an inside diameter of $56 \mathrm{~mm}$ and an outside diameter of 64 $\mathrm{mm}$. A plug was inserted into the rostral end of the tube and held in position by nylon screws. The inside of this rostral plug was fabricated in a cone shape to accommodate the head of the rat. A $1 \mathrm{~cm}$ hole was bored in this plug at the apex of this cone to allow fresh breathing air. A second plug was inserted into the caudal end of the tube and could be adjusted to restrain the movement of the rat. A hole in the center of this plug accommodated the rat's tail. When in the tube, the rat was almost completely immobilized. Individual restrained rats were carried from the animal facility to the superconducting magnets $(\sim 100 \mathrm{~m}$ distance). The restrained rat was inserted into the bottom of the vertical bore of the magnet and raised until the head of the rat was in the center of the magnetic field. Rats remained in the bore of the magnet for 1-30 min.

To control restraint and handling, sham-exposed rats were allowed 10 $\min$ to access $\mathrm{G}+\mathrm{S}$ and were then inserted in identical restraining tubes. The sham-exposed rats were vertically inserted into an opaque polyvinylchloride (PVC) pipe with dimensions and conditions (sound, light, and temperature) similar to those of the magnet bore. One magnetexposed rat and one sham-exposed rat were conditioned in parallel time.

Magnets. The magnet exposures were done in one of two superconducting magnets with vertical bores designed for biochemical nuclear magnetic resonance (NMR) studies. The $7 \mathrm{~T}$ magnet was an Oxford Instruments (Concord, MA) D 15,000/19/19 $300 \mathrm{MHz}$ magnet with a fixed field strength of $7 \mathrm{~T}$ and a $89 \mathrm{~mm}$ bore. The $14 \mathrm{~T}$ magnet was a 600 $\mathrm{MHz}$ Bruker Cryo magnet with an $89 \mathrm{~mm}$ bore and a fixed field strength of $14.1 \mathrm{~T}$. Both magnets contained shim magnets extending along the magnet bore for approximately $\pm 15 \mathrm{~cm}$ from the magnet core, which were used to stabilize the magnetic field and give a central core field of uniform strength. The magnetic fields in both magnets were orientated vertically so that the positive pole was at the top of the magnet. The magnets were operated without radiofrequency pulses, so rats were exposed to only static magnetic fields.

Locomotor activity. At the conclusion of magnet or sham exposure, each rat was carried back to the animal facility while still restrained. The rostral plug of the restraining tube was removed, and the rat was allowed to emerge from the tube into an open Plexiglas cage $(37 \mathrm{~cm}$ wide $\times 47 \mathrm{~cm}$ long $\times 20 \mathrm{~cm}$ high). The floor of this open-field cage was covered with cob bedding. The locomotor behavior of each rat was recorded on videotape for $2 \mathrm{~min}$ after release into the cage. (Most rats exhibited locomotor effects of the magnetic field for $<1 \mathrm{~min}$; only one rat in the present study showed an effect for $>2 \mathrm{~min}$. Thus, $2 \mathrm{~min}$ of recording captured most of the phenomenon of interest.) The rat was then returned to its home cage. The videotapes were scored later by an observer blind to the rats' treatment. Instances of tight-circling behavior and rearing behavior (one or both forepaws on the side of the cage) were quantified. Rats were scored as "circling" if they moved continuously around a full circle with a diameter less than length of the rats body (i.e., with nose almost touching the end of the tail) Partial circles or circles interrupted by stationary pauses were not counted.

Preference tests. To test for magnetic field-induced CTA, a series of 24 hr, two bottle preference tests was initiated on the day after the last conditioning trial. Two bottles were placed on the cages, one containing the $\mathrm{G}+\mathrm{S}$ solution and the other containing distilled water. Fluid con- sumption was measured every $24 \mathrm{hr}$ and a preference score was calculated as a ratio of $\mathrm{G}+\mathrm{S}$ solution to total fluid consumption.

The preference tests were continued for 5-9 postconditioning days. Because $G+S$ access during the preference tests was not paired with magnet exposure, the preference tests constituted extinction trials. A CTA was considered extinguished when the average $G+S$ preference of magnet-exposed rats was not different from the average preference of sham-exposed rats.

In summary, measurements were made of four dependent variables: (1) visual scoring of the locomotor behavior of the rats immediately after the first magnet or sham exposure, as a test of the immediate effects on activity; (2) consumption of the $\mathrm{G}+\mathrm{S}$ solution during the $10 \mathrm{~min} \mathrm{CS}$ period on the second and third conditioning days as a one bottle test of CTA during acquisition (experiment 2); (3) preference score for $G+S$ versus water on the first day of postconditioning preference testing as a two bottle test of CTA expression; and (4) the number of days of preference testing required for extinction of the CTA.

Statistical analysis. Significant effects were determined using one- or two-way ANOVA. Post hoc comparisons were made using Fisher's least significant difference (FLSD) test, $t$ test, or orthogonal comparison as indicated. As binomial variables, the significant presence or absence of circling and rearing was tested using the $\chi^{2}$ test.

Experiment 1: single pairing of $G+S$ and magnet exposure. A total of 65 rats were housed and placed on the water-deprivation schedule as described above. On the first conditioning day, all rats had access to the $\mathrm{G}+\mathrm{S}$ solution for $10 \mathrm{~min}$. Immediately after this drinking period, rats were placed in the restraining tubes and individually inserted head-up into either the core of the $7 \mathrm{~T}$ magnet for $30 \mathrm{~min}$ (experiment $1 \mathrm{a} ; n=9$ ) or the core of the $14 \mathrm{~T}$ magnet for a varying duration of exposure (experiment $1 \mathrm{~b})$. One group of rats $(n=6)$ was inserted and immediately removed from the core of the $14 \mathrm{~T}$ magnet and thus received minimal exposure to the magnetic field $(0 \mathrm{~min})$. Additional rats were exposed to the $14 \mathrm{~T}$ magnetic field for $1,5,10,20$, or $30 \mathrm{~min}$ ( $n=6-14$ /group). Control, sham-exposed rats were inserted into the sham PVC tube for 30 $\min (n=5)$.

After being removed from the magnet (or sham magnet), the rostral plug was removed from the restraining tube and the rat was allowed to emerge into the open-field testing chamber. Locomotor behavior was recorded on videotape for $2 \mathrm{~min}$. The rats were then returned to their home cage and given ad libitum access to water. On the following day, the first $24 \mathrm{hr}$, two bottle preference test between $\mathrm{G}+\mathrm{S}$ and water began. The $24 \mathrm{hr}$ preference tests were continued for 7-9 d.

Experiment 2: three pairings of $G+S$ and magnet exposure. A total of 35 rats were housed and placed on the water-deprivation schedule as described above. On the first conditioning day, all of the rats had access to the $\mathrm{G}+\mathrm{S}$ solution for $10 \mathrm{~min}$. Immediately after this drinking period, half of the rats were inserted into either the $7 \mathrm{~T}$ magnet $(n=8)$ or the $14 \mathrm{~T}$ magnet $(n=10)$ and remained at the magnet core for $30 \mathrm{~min}$. The remaining rats were inserted into the sham PVC tube for $30 \mathrm{~min}$. After being removed from the magnet (or sham magnet), the rostral plug was removed from the exposure chamber and the rat was allowed to emerge from the restraining chamber into the open-field testing chamber. Locomotor behavior was recorded on videotape for $2 \mathrm{~min}$. The rats were then returned to their home cage and remained on water deprivation until the following day, when the second conditioning trial was given. This procedure was repeated for a third day of conditioning. After the third pairing of $\mathrm{G}+\mathrm{S}$ and magnet (or sham) exposure, the locomotion behavior was measured and the animals were returned to their home cages and given ad libitum access to water. On the following day, the first $24 \mathrm{hr}$, two bottle preference test between $\mathrm{G}+\mathrm{S}$ and water began. These $24 \mathrm{hr}$ preference tests continued for $10 \mathrm{~d}$.

Experiment 3: orientation within the magnet. In the course of experiments 1 and 2, it was found that rats exposed to the 14 T magnetic field for 30 min reliably exhibited circling behavior when released from restraint, whereas approximately half of the rats exposed to the $7 \mathrm{~T}$ magnet or exposed for shorter periods in the $14 \mathrm{~T}$ magnet exhibited circling behavior. If a rat did circle, however, the rotation of the rat was always in the counterclockwise direction. All of these rats had been placed in the vertical bore of the magnets head-up, facing the positive pole of the magnetic 
Table 1. Effects of magnetic field exposure on locomotor activity

\begin{tabular}{|c|c|c|c|c|c|c|}
\hline Field & Duration & Number of rats & \% Circling & Mean circles & \% Rearing & Mean rears \\
\hline \multicolumn{7}{|c|}{ Experiments $1 \mathrm{a}$ and 2} \\
\hline $0 \mathrm{~T}$ & $30 \mathrm{~min}$ & 22 & 5 & 1 & 91 & $3.8 \pm 0.5$ \\
\hline $7 \mathrm{~T}$ & $30 \mathrm{~min}$ & 17 & 47 & $2.4 \pm 0.6^{*}$ & 59 & $2.8 \pm 0.9^{*}$ \\
\hline $14 \mathrm{~T}$ & $30 \mathrm{~min}$ & 17 & 71 & $2.6 \pm 0.4^{*}$ & 0 & $0^{* \dagger}$ \\
\hline \multicolumn{7}{|l|}{ Experiment $1 \mathrm{~b}$} \\
\hline $14 \mathrm{~T}$ & $0 \min$ & 6 & 0 & $0 \pm 0$ & 100 & $4.8 \pm 0.8$ \\
\hline $14 \mathrm{~T}$ & $1 \mathrm{~min}$ & 6 & 0 & $0 \pm 0$ & 100 & $1.8 \pm 0.7^{*}$ \\
\hline $14 \mathrm{~T}$ & $5 \min$ & 8 & 50 & $5.0 \pm 2.5$ & 67 & $2.3 \pm 0.5^{*}$ \\
\hline $14 \mathrm{~T}$ & $10 \min$ & 14 & 57 & $2.9 \pm 0.7$ & 43 & $2.2 \pm 0.5^{*}$ \\
\hline $14 \mathrm{~T}$ & $20 \mathrm{~min}$ & 8 & 63 & $2.8 \pm 0.5$ & 13 & $1^{*}$ \\
\hline $14 \mathrm{~T}$ & $30 \mathrm{~min}$ & 17 & 71 & $2.6 \pm 0.4$ & 0 & $0^{*}$ \\
\hline \multicolumn{7}{|l|}{ Experiment 3} \\
\hline oTDown & $30 \mathrm{~min}$ & 12 & 0 & 0 & 83 & $4.9 \pm 1.1$ \\
\hline 14 T Down & $30 \mathrm{~min}$ & 15 & 73 & $6.1 \pm 2.7$ & 33 & $2.0 \pm 0.3^{*}$ \\
\hline 14 T Up & $30 \mathrm{~min}$ & 13 & 77 & $8.4 \pm 2.5$ & 54 & $1.6 \pm 0.3^{*}$ \\
\hline
\end{tabular}

Locomotor activity of rats after magnetic field exposure was scored for tight circling and rearing by a blind observer from videotape records. Rats were sham exposed ( $0 \mathrm{~T}$ ) or exposed to 7 or $14 \mathrm{~T} \mathrm{magnetic} \mathrm{fields} \mathrm{for} 0$ - $30 \mathrm{~min}$. Shown are the number of rats tested, the percentage of rats displaying circling or rearing, and the mean \pm SEM number of circles or rears counted for those rats that showed any circling or rearing (i.e. non-zero means). In experiments $1 \mathrm{a}$ and 2 , rats were exposed head-up to a 7 or $14 \mathrm{~T}$ magnetic field for $30 \mathrm{~min}$; both exposures induced circling and suppressed rearing. In experiment $1 \mathrm{~b}$, rats were exposed head-up to $14 \mathrm{~T}$ for 0 - 30 min; significant circling was induced by $\geq 5$ min of exposure, and rearing was significantly suppressed after $\geq 10$ min of exposure. In experiment 3 , rats were restrained in either the head-up or head-down orientation within a $14 \mathrm{~T}$ magnetic field for 30 min. Circling was induced and rearing was suppressed in both groups, but the direction of circling depended on the rats' orientation within the field (Fig. 1).

field. The purpose of this experiment was to test the hypothesis that the circling behavior was determined by the rat's orientation within the magnet.

Acute behavioral effects. Eighteen male rats were housed as described above but allowed ad libitum access to water. Four rats were exposed within the vertical bore of the $14 \mathrm{~T}$ magnet head-up; eight were exposed head-down, pointing toward the negative pole of the magnet. The other six rats were sham-exposed head-down in the vertical PVC tube. Locomotor activity in the open-field test was recorded immediately after exposure.

CTA expression. To compare the magnitudes of CTAs induced by head-up or head-down exposure to the $14 \mathrm{~T}$ magnet, an additional 24 rats were housed and maintained on a water deprivation schedule as above. On conditioning day, rats were allowed 10 min of access to $G+S$ and then restrained and exposed within the vertical bore of the magnet in either the head-up orientation $(n=6)$ or the head-down orientation $(n=8)$ for $30 \mathrm{~min}$. The remaining rats were sham-exposed head-down for $30 \mathrm{~min}$. Locomotor activity in the open-field test was recorded immediately after exposure. The rats were then returned to their home cage, and their water bottle was returned. On the following day, the first two bottle preference test between $\mathrm{G}+\mathrm{S}$ and water was initiated. These $24 \mathrm{hr}$ preference tests were continued for $10 \mathrm{~d}$.

\section{Results}

\section{Locomotor effects}

The effects of exposure to magnetic fields on the locomotor behaviors of tight circling and rearing are summarized in Table 1.

\section{Experiments 1a and 2: exposure to 7 or $14 \mathrm{~T}$ magnetic field for $30 \mathrm{~min}$}

Open-field locomotor activity was scored for tight circling and rearing after the first $30 \mathrm{~min}$ exposure to 0 (sham), 7 , or $14 \mathrm{~T}$ in experiments 1 and 2. Significantly more magnet-exposed rats exhibited circling locomotor behavior; only one of the shamexposed rats exhibited any circling behavior ( $\chi^{2}$ test; see Table 1$)$. All rats that circled moved in a counterclockwise direction (Fig. $1 A)$. Rearing was also significantly reduced in magnet-exposed rats $\left(\chi^{2}\right.$ test $)$.

Experiment 1b: duration of exposure to $14 \mathrm{~T}$ magnetic field Locomotor activity was scored after $0-30 \mathrm{~min}$ restraint within the $14 \mathrm{~T}$ magnet. There was a significant effect of the duration of exposure to the $14 \mathrm{~T}$ magnetic field on the number of rats circling

\section{A. Head-Up}

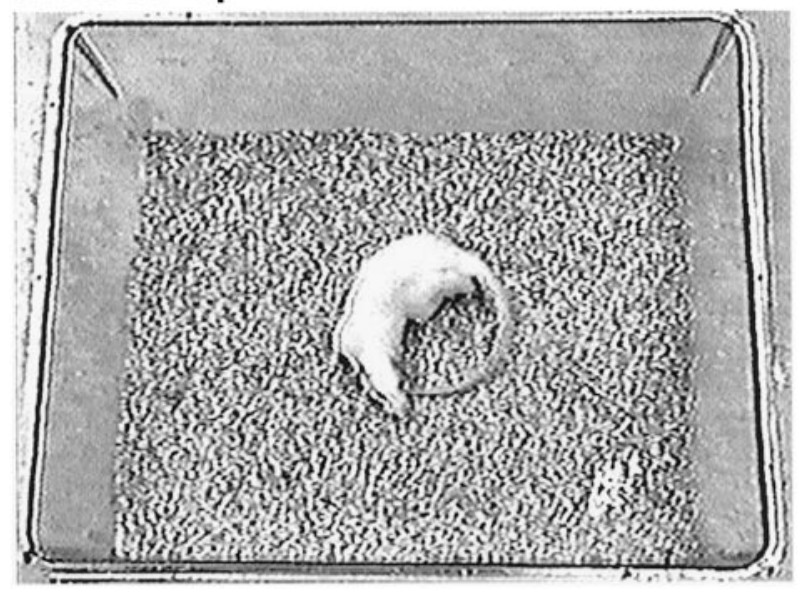

\section{B. Head-Down}

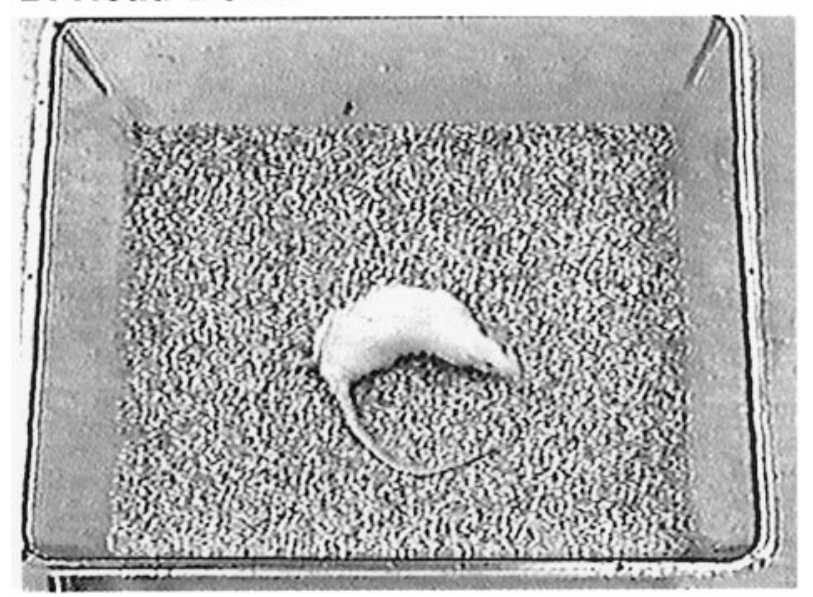

Figure 1. Tight-circling activity induced by magnetic field exposure. Rats were restrained for 30 min within the bore of a $14 \mathrm{~T}$ magnet in either head-up orientation $(A)$ or head-down orientation $(B)$. On release from restraint, rats oriented head-up circled counterclockwise, whereas rats oriented head-down circled clockwise. 
and rearing (as determined by $\chi^{2}$ test). Counterclockwise circling was induced by exposures of $\geq 5 \mathrm{~min}$; rearing was significantly reduced after only $1 \mathrm{~min}$ of exposure.

Experiment 3: orientation within the magnetic field

Magnetic field exposure significantly induced circling in rats regardless of orientation ( $\chi^{2}$ test), but the direction of rotation after $14 \mathrm{~T}$ exposure was dependent on the orientation of rats within the magnetic field. Most 14 T magnet-exposed rats showed circling: circling rats exposed head-up turned exclusively counterclockwise (Fig. $1 A$ ); circling rats exposed head-down turned exclusively clockwise (Fig. 1B). Sham-exposed rats did not circle. There was some rearing behavior in all three groups, but the average number of rears was significantly decreased in the two magnet-exposed groups compared with the sham-exposed group $\left(F_{(2,37)}=3.8 ; p<0.05\right)$.

\section{Conditioned taste aversion effects}

Experiment 1a: single pairing of $G+S$ with 7, 9.4, or $14 T$

Rats were given a single pairing of $\mathrm{G}+\mathrm{S}$ intake with $30 \mathrm{~min}$ of exposure to 0 (sham), 7 , or $14 \mathrm{~T}$ (rats from the 30 min group of experiment 1b). For purposes of comparison, data collected in the previous study after a single pairing of $G+S$ with 30 min of exposure to 9.4 T were also included in the analysis (Nolte et al., 1998). Magnetic field strength had a significant effect on $G+S$ preference on the first test day after pairing $\left(F_{(3,25)}=3.0 ; p<\right.$ 0.05 ) (Fig. 2A). Post hoc tests showed that rats exposed to $14 \mathrm{~T}$ after $G+S$ intake had a significantly lower preference for $G+S$ compared with sham-exposed rats, but the preferences of rats exposed to 7 or $9.4 \mathrm{~T}$ were not significantly different from either sham-exposed rats or $14 \mathrm{~T}$-exposed rats. Therefore, a single pairing of $\mathrm{G}+\mathrm{S}$ with a $30 \mathrm{~min}$ exposure to $14 \mathrm{~T}$ was sufficient to induce a CTA to $\mathrm{G}+\mathrm{S}$, but a single pairing with 7 or $9.4 \mathrm{~T}$ was at or below threshold for inducing a CTA. The $\mathrm{G}+\mathrm{S}$ preference of rats exposed to $14 \mathrm{~T}$ was not significantly different from shamexposed rats by the third testing day (Fig. $2 B$ ).

\section{Experiment 1b: duration of exposure to magnetic field}

Rats were given a single pairing of $\mathrm{G}+\mathrm{S}$ intake with $0-30 \mathrm{~min}$ of exposure to the $14 \mathrm{~T}$ magnetic field. On the first day of two bottle testing, there was a significant effect of duration of exposure on $\mathrm{G}+\mathrm{S}$ preference $\left(F_{(5,42)}=4.97 ; p<0.005\right)$ (Fig. $\left.3 A\right)$. Post hoc analysis (FLSD) showed that rats that received $\geq 1$ min of exposure had a significantly lower preference for $\mathrm{G}+\mathrm{S}$ compared with rats that received no exposure ( $p$ values of $<0.01$ ). With subsequent $24 \mathrm{hr}$, two bottle tests of $\mathrm{G}+\mathrm{S}$ preference, there was a significant interaction of extinction day and duration of exposure $\left(F_{(5,30)}=1.67 ; p<0.05\right)$ (Fig. $\left.3 B\right)$. Thus, a single pairing of $\mathrm{G}+\mathrm{S}$ with $\geq 1 \mathrm{~min}$ of exposure to $14 \mathrm{~T}$ was sufficient to induce a CTA.

Experiment 2: three pairings of $G+S$ with 7 or $14 T$

Rats were given three pairings of $G+S$ intake with 30 min of exposure to 0 (sham), 7, or $14 \mathrm{~T}$. For purposes of comparison, data collected in the previous study after three pairings of $G+S$ with $30 \mathrm{~min}$ of exposure to $9.4 \mathrm{~T}$ were included in the analysis (Nolte et al., 1998).

\section{Intake during conditioning}

Because rats had only 10 min access to fluid during the 3 conditioning days, differences in intake were not large during conditioning. Some significant decreases were detected by $t$ test in some magnet-exposed groups, however. There was no significant difference in intake of $\mathrm{G}+\mathrm{S}$ between $7 \mathrm{~T}$-exposed and the sham-
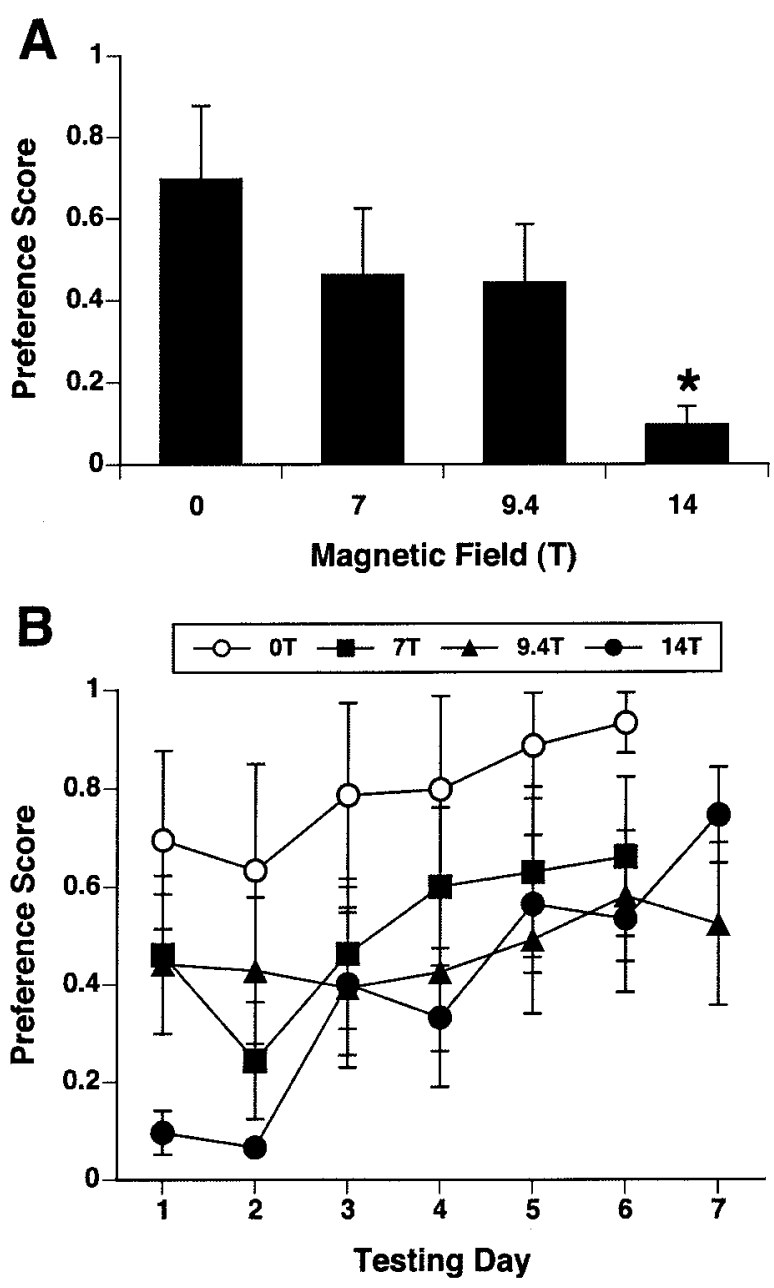

Figure 2. CTAs induced by a single pairing of $\mathrm{G}+\mathrm{S}$ intake with a 30 min restraint within magnetic fields of different strengths. For the $24 \mathrm{hr}$ two bottle preference test after the pairing of $\mathrm{G}+S$ with magnetic exposure $(A)$, a significant $(T A$ against $\mathrm{G}+S$ was observed only after pairing with $14 \mathrm{~T}$ exposure. The CTA extinguished after $3 \mathrm{~d}$ of two bottle preference tests $(B)$. ${ }^{*} p<0.05$ versus $0 \mathrm{~T}$ (sham) exposure. Data for $9.4 \mathrm{~T}$ exposure are replotted from Nolte et al. (1998).

exposed rats on any of the 3 conditioning days (Fig. $4 A$ ). $\mathrm{G}+\mathrm{S}$ intake was not significantly different between the $9.4 \mathrm{~T}$-exposed and sham-exposed rats on the first and second conditioning days, but intake was significantly lower in $9.4 \mathrm{~T}$-exposed rats on the third conditioning day (Fig. $4 \mathrm{~B}$ ). In contrast, the $\mathrm{G}+\mathrm{S}$ intake was significantly lower in $14 \mathrm{~T}$-exposed than sham-exposed rats on the second and third conditioning days (Fig. 4C).

\section{First two bottle test after three pairings}

Magnetic field strength had a significant effect on $G+S$ preference on the first two bottle test day after pairing $\left(F_{(3,28)}=9.4 ; p<\right.$ 0.0005 ) (Fig. 5A). A graded response was found by orthogonal comparison, such that $G+S$ preference was significantly decreased compared with sham-exposed rats after three pairings with $7 \mathrm{~T}$ and significantly decreased compared with $7 \mathrm{~T}$ after pairings with 9.4 or $14 \mathrm{~T}$.

\section{Extinction after three pairings}

Extinction of the magnet-induced CTAs across $9 \mathrm{~d}$ of two bottle preference tests was analyzed at each field strength by repeated, two-way ANOVAs with time and treatment as factors, and by post hoc analysis by FSLD. After three pairings at $7 \mathrm{~T}$, an extinction 

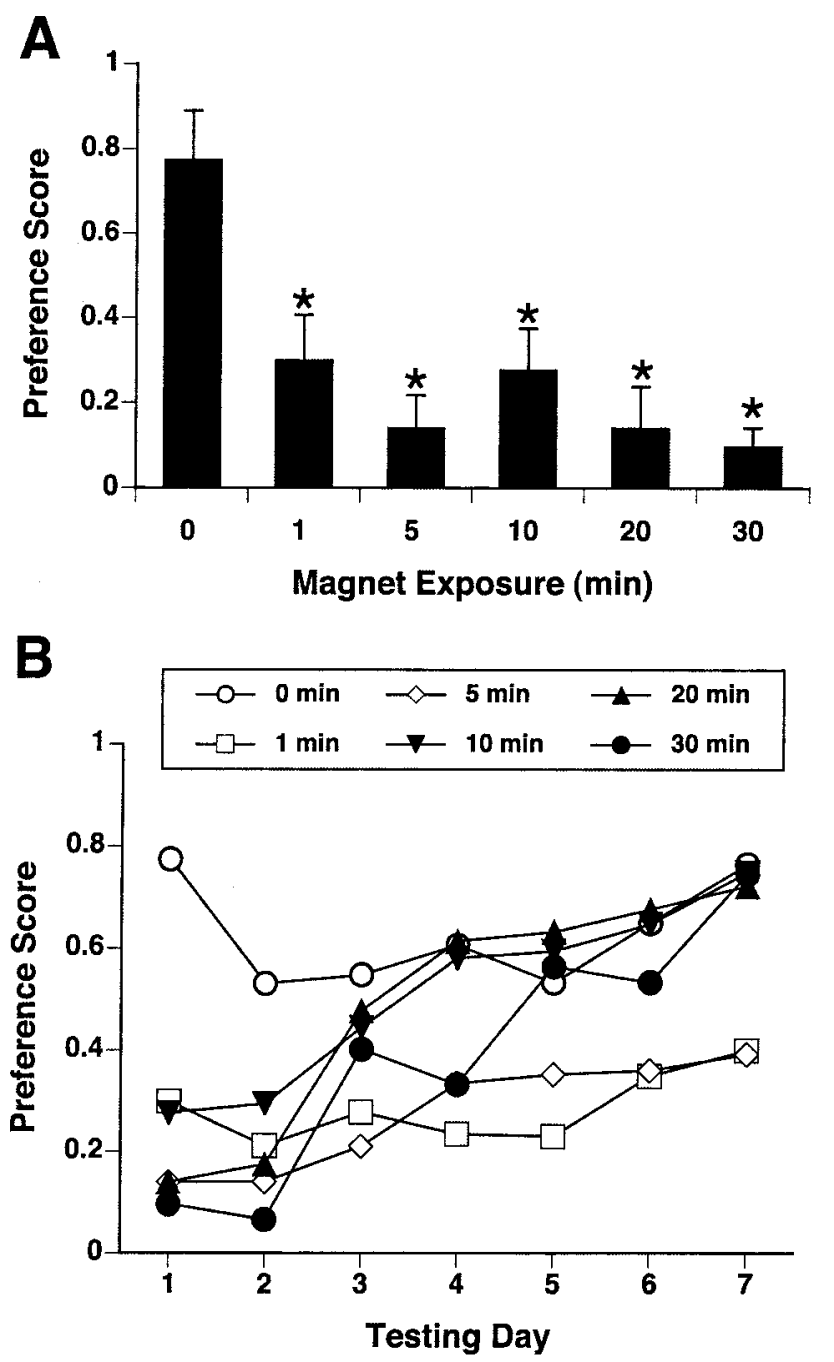

Figure 3. CTAs induced by a single pairing of $G+S$ intake with restraint within 14 T magnetic fields for $0-30 \mathrm{~min}$. Significant (TA was observed after $\geq 1 \mathrm{~min}$ of exposure to the magnetic field on the first $24 \mathrm{hr}$ two bottle preference test $(A)$; the CTAs persisted for several days of preference testing $(B) .{ }^{*} p<0.05$ versus 0 min exposure.

effect was found $\left(F_{(8,112)}=3.19 ; p<0.005\right)$ (Fig. $\left.5 B\right)$. G+S preference was significantly decreased in $7 \mathrm{~T}$-exposed rats compared with sham-exposed rats on days 1 and 2.

After three pairings at $9.4 \mathrm{~T}$, there was a significant interaction of extinction day and treatment $\left(F_{(8,112)}=5.96 ; p<0.001\right)$ (Fig. $5 C) . \quad G+S$ preference was significantly decreased in 9.4 T-exposed rats compared with sham-exposed rats for days $1-8$, but not on day 9. Similarly, after three pairings at $14 \mathrm{~T}$, there was a significant interaction of extinction and treatment $\left(F_{(8,112)}=\right.$ 4.06; $p<0.001$ ) (Fig. 5D). G+S preference was significantly decreased in $14 \mathrm{~T}$-exposed rats compared with sham-exposed rats for days $1-8$, but not on day 9 .

Experiment 3: orientation within magnetic field

Both groups of rats exposed to the $14 \mathrm{~T}$ magnet in head-up or head-down orientation after $\mathrm{G}+\mathrm{S}$ access formed significant CTAs that extinguished by the third postconditioning test day $\left(F_{(5,90)}=9.49 ; p<0.001\right)$ (Fig. 6). The $\mathrm{G}+\mathrm{S}$ preferences were not different between the two magnet-exposed groups on the first day, but both magnet-exposed groups showed significantly lower $\mathrm{G}+\mathrm{S}$ preference than head-down, sham-exposed rats. Thus, re-

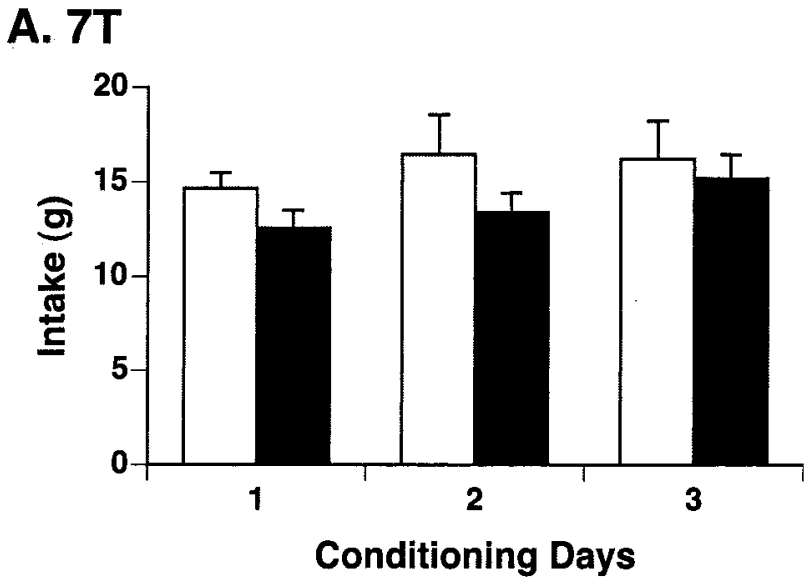

B. 9.4T
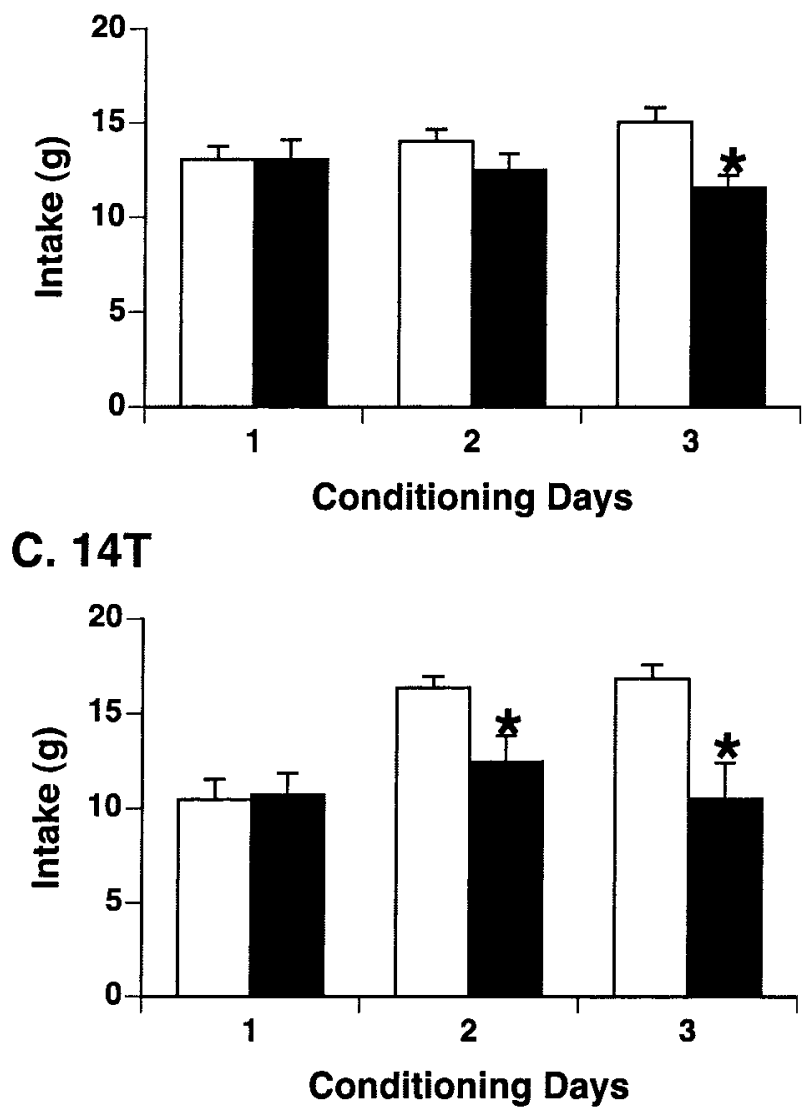

Figure 4. Acquisition of CTAs across three pairings of $G+S$ with 30 min restraint within 7 , 9.4, or $14 \mathrm{~T}$ magnetic fields. Intake during $10 \mathrm{~min}$ access to $\mathrm{G}+\mathrm{S}$ was not different between sham- and $7 \mathrm{~T}$-exposed rats across the 3 conditioning days $(A)$. Rats exposed to $9.4 \mathrm{~T}$ decreased intake compared with sham-exposed rats on the third day of conditioning (i.e., after two pairings; $B$ ). Rats exposed to $14 \mathrm{~T}$ decreased intake on the second day of conditioning (i.e., after one pairing; (). ${ }^{*} p<0.05$ versus sham-exposed rats. Data for $9.4 \mathrm{~T}$ exposure are replotted from Nolte et al. (1998).

straint within the $14 \mathrm{~T}$ magnetic field produced equivalent CTAs regardless of the rat's orientation within the magnet.

\section{Discussion}

In this study, exposure to high-strength static magnetic fields had acute effects on the locomotor behavior of rats, and a CTA was induced when a novel palatable solution was paired with exposure to the magnetic fields. Both 7 and $14 \mathrm{~T}$ magnets induced 
A.

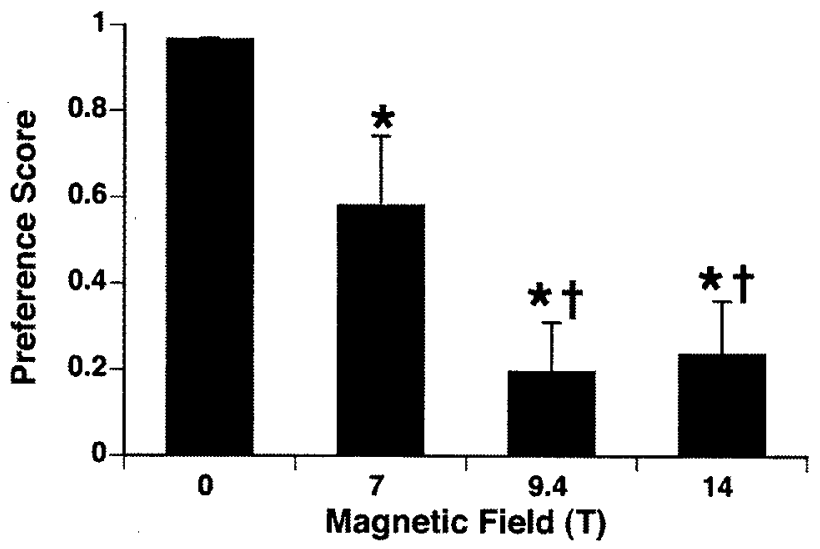

C. $9.4 \mathrm{~T}$

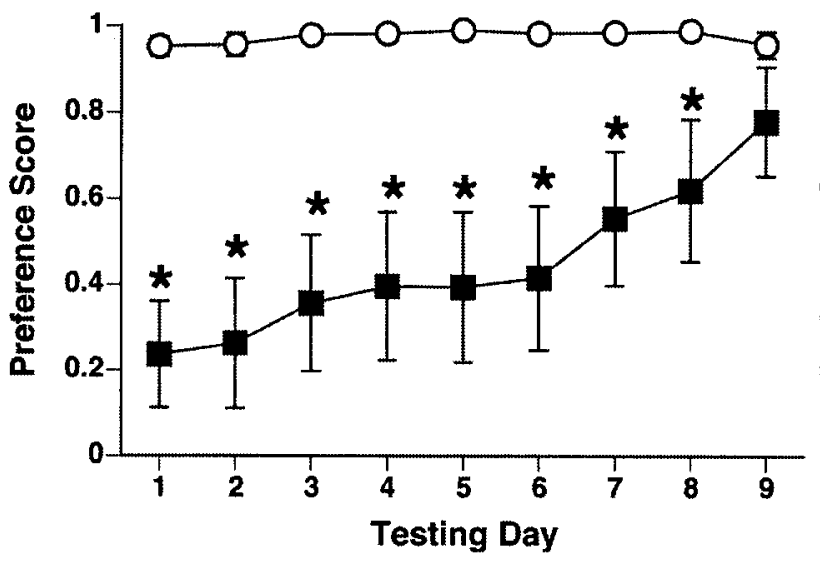

B. 7 T

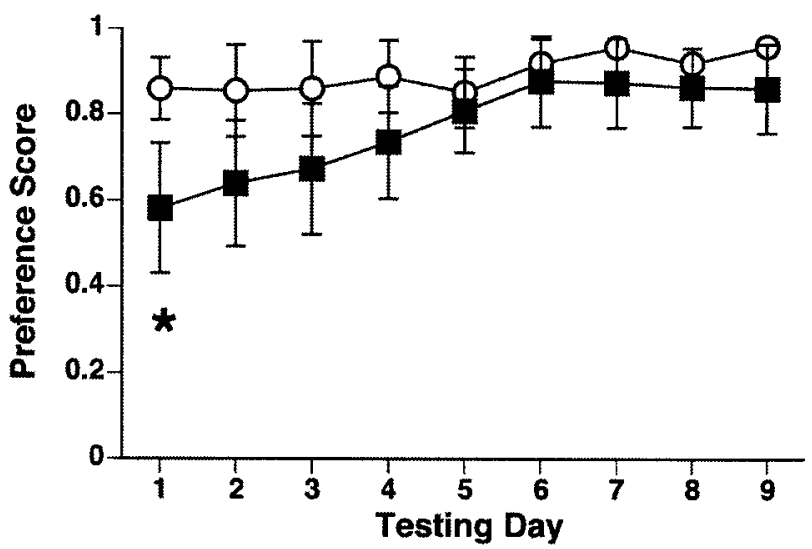

D. $14 \mathrm{~T}$

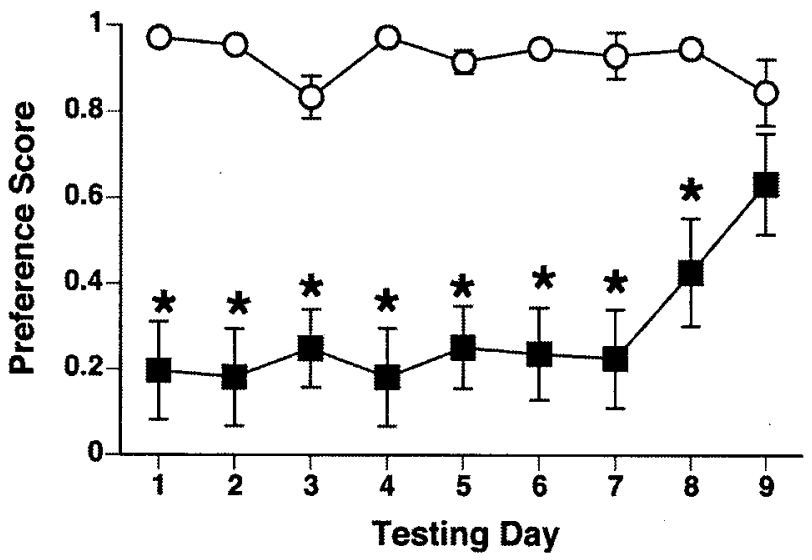

Figure 5. CTAs induced by three pairings of $\mathrm{G}+\mathrm{S}$ intake with restraint within $7,9.4$, or $14 \mathrm{~T}$ magnetic fields for $0-30 \mathrm{~min}$. Significant CTAs were observed in all magnet-exposed rats on the first $24 \mathrm{hr}$ two bottle preference test $(A)$. Over subsequent $24 \mathrm{hr}$ two bottle test days, the (TA of $7 \mathrm{~T}$-exposed rats extinguished on the second day $(B)$, whereas the (TAs of $9.4 \mathrm{~T}$ - and $14 \mathrm{~T}$-exposed rats persisted for $8 \mathrm{~d}$ of preference testing $(C, D) .{ }^{*} p<0.05$ versus sham-exposed rats; ${ }^{\dagger} p<0.05$ versus 7 T-exposed rats. Data for $9.4 \mathrm{~T}$ exposure are replotted from Nolte et al. (1998).

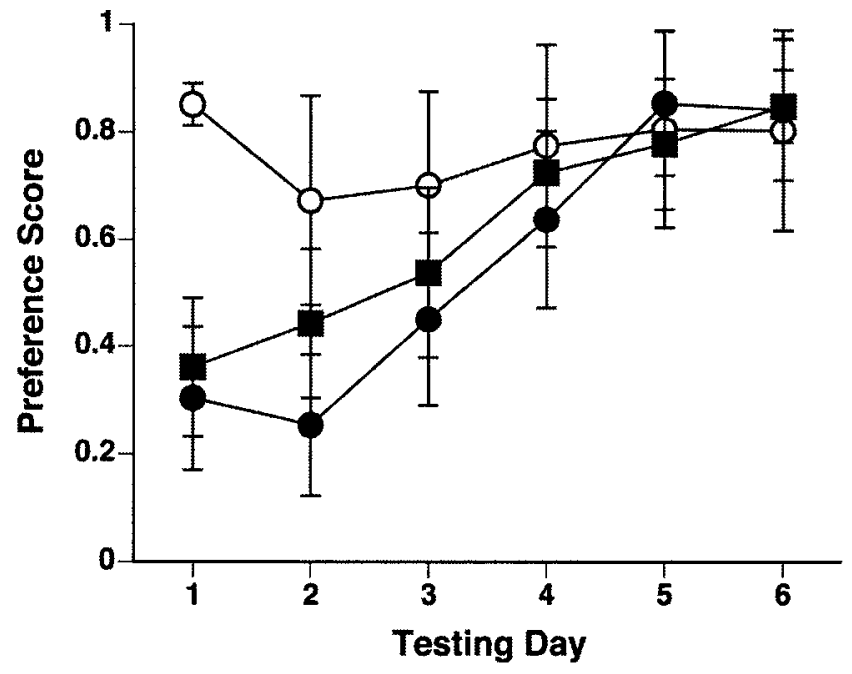

Figure 6. CTAs induced by a single pairing of $\mathrm{G}+\mathrm{S}$ with $14 \mathrm{~T}$ restraint in head-up or headdown orientations. After 10 min of access to $G+S$, rats were restrained in either a head-up orientation (black squares) or head-down orientation (black circles) for 30 min within the $14 \mathrm{~T}$ magnetic field. Although rats circled in opposite directions on release from restraint (with head-up rats circling counterclockwise and head-down rats circling clockwise), there was little difference in the CTA acquisition of the two groups. Control rats were sham-exposed while head-down for $30 \mathrm{~min}$ (white circles). tight-circling locomotor activity and suppressed rearing, and both magnets induced CTA. The direction of circling (but not the magnitude of CTA) was dependent on the rat's orientation within the field. By quantifying both locomotor activity and CTA expression, and including data from a previous report (Nolte et al., 1998), a graded response to field strength was found with rank ordering of $14>9.4>7 \mathrm{~T}$. Furthermore, the threshold of the magnetic field exposure sufficient for CTA acquisition after one pairing with $\mathrm{G}+\mathrm{S}$ was found to be close to one pairing of $30 \mathrm{~min}$ at 7 or $9.4 \mathrm{~T}$, and between one pairing of 0 and $1 \mathrm{~min}$ at $14 \mathrm{~T}$. Thus the behavioral effects on rats of magnetic fields are graded and specific to field intensities and durations of exposure.

We have now observed circling and CTA acquisition on different days on three magnets from two different manufacturers. The replication of the qualitative effects of magnetic field exposure is significant: the behavioral effects appear to be caused by the presence of a high-strength magnetic field and not by an artifact of specific equipment or a specific test session. Similarly, the observation of graded effects is significant, because the three magnets have variable field strengths but are otherwise similar in terms of materials, circuitry, coolants, interior temperature, and noise, for example.

\section{Locomotor effects}

The magnetic fields induced circling and suppressed rearing proportional to field strength. These locomotor effects are compara- 
ble with the circling seen after unilateral hemilabyrinthectomy (Kaufman et al., 1999) and the suppression of rearing seen after horizontal rotation (Ossenkopp et al., 1994). The tight circling seen after magnetic field exposure may also be an indirect result of activation of other motor systems. Unilateral lesions within the striatum, ascending dopaminergic pathways, cerebellum, or vestibular nuclei can induce spontaneous circling or susceptibility to circling after drug or vestibular stimulation (Shima, 1984). Because of the complicated reciprocal connections of the vestibular and motor systems, it is impossible to localize the site of magnetic field action from the intact rat's behavioral response alone.

Rats did not always circle on release from a magnetic field, although the proportion of rats that circled increased with the strength of the magnetic field. The variation in the initiation of circling and the number of times an individual rat circles may reflect variation in the susceptibility of individual rats or variation in experimental parameters.

Although the magnitude of circling behavior is variable, the direction of circling has been completely consistent across all experiments to date. Furthermore, the direction was determined by the rats' orientation within the field. Not all rats circled on release, but if a rat did circle, it always circled counterclockwise if restrained head-up or clockwise if restrained head-down. Because the field strength of superconducting NMR magnets is fixed and their polarity is difficult to reorient, we reoriented the rat by inversion within the vertical bore of the magnet. Therefore, we cannot determine whether the direction of circling is attributable to the relative polarity of the magnetic field or the headup-head-down orientation of the rat. Future experiments in large-bore, resistive magnets in which the rat's body and the polarity of the magnet can be arbitrarily oriented will help define the parameters of this effect.

The consistent effects of the magnetic field on the direction of circling strongly suggest unilateral or asymmetrical stimulation of the inner ear, vestibular nuclei, or motor pathways. Furthermore, the correlation of circling direction with the rat's orientation within the field suggests an inherent asymmetry in the interaction of the magnetic field with the vestibulomotor system of the rat. Although the mechanism and site of action is obscure, the engagement of the vestibulomotor system by magnetic fields suggests candidate neural sites involved in the rat's detection of the magnetic field. In fact, c-Fos immunohistochemistry in the rat brainstem $1 \mathrm{hr}$ after $9.4 \mathrm{~T}$ magnetic field exposure has revealed activation of vestibular and visceral nuclei (Snyder et al., 2000). We cannot, however, rule out effects of the magnetic field on the basal ganglia-midbrain dopaminergic system (Shima, 1984) or prefrontal cortex (Nakamura-Palacios et al., 1999) that can also lead to circling behavior and indirectly to vestibular activation. The use of females (Choleris et al., 2000) or other rodent strains (Cransac et al., 1997; Richter et al., 1999) that are more sensitive to vestibular stimulation may be useful.

\section{Taste aversion}

CTA learning has been used to reveal the detection and responsiveness of animals to many pharmacological, vestibular, or radiation treatments. It has a number of advantages for the exploration of the effects of magnetic fields. The rate of CTA acquisition, the magnitude of the CTA, and rate of extinction can be used as measures of the strength of the detected US.

Because CTA learning tolerates a long delay (Smith and Roll, 1967) between presentation of the conditioned stimulus (the taste) and the unconditioned treatment (magnet exposure) and is largely insensitive to environmental context (Garcia and Koel- ling, 1966), the magnetic field exposure could be separated in time and space from the taste stimulus. Because the expression of a CTA can be measured for days after acquisition, the graded effects of the magnetic fields could be measured by two bottle preference tests in extinction trials independent of any shortterm effects of the magnetic field on the rat. Finally, because of the rat's sensitivity to taste-toxin associations, a CTA can often reveal the rat's ability to detect an unconditioned stimulus even in the absence of any other observable behavioral effect. Thus, rats were shown to acquire a CTA against $\mathrm{G}+\mathrm{S}$ after only a 1 min exposure to a $14 \mathrm{~T}$ magnetic field.

As with locomotor effects, the magnitude of the CTA was related to the intensity of the magnetic field. Pairing the G+S solution with one $30 \mathrm{~min}$ exposure to the $7 \mathrm{~T}$ magnet was not sufficient to induce a significant CTA; in the previous study, one 30 min exposure to a $9.4 \mathrm{~T}$ magnet was sufficient to induce a weak but significant CTA (Nolte et al., 1998). Thus we have identified a minimal intensity of the magnetic field sufficient for CTA induction under our conditions. However, different behavioral effects observed under different conditions may reveal different thresholds of sensitivity to magnetic fields. Thus, the $7 \mathrm{~T}$ magnet was not without effect: circling was occasionally observed after 7 $\mathrm{T}$ exposure, and a CTA could be acquired with three pairings of $\mathrm{G}+\mathrm{S}$ with 30 min of exposure to the $7 \mathrm{~T}$ magnet.

It is important to emphasize that rats will learn a CTA not only to toxic, aversive, or nauseating stimuli but will also avoid tastes paired with many treatments that are not physically toxic (e.g., rotation) or obviously aversive [e.g., orexigenic drugs (Stephan et al., 1999) or self-administered drugs of abuse (Parker, 1995)]. Furthermore, although rats will easily form taste aversions to treatments that induce nausea in humans, many treatments that do not induce nausea in humans will induce CTA in rats. Therefore, we cannot conclude that magnetic fields are aversive, toxic, or malaise inducing just because rats acquire a CTA after magnetic field exposure. Nonetheless, because both magnetic fields and vestibular stimulation by rotation or labyrinthectomy can induce circling behavior and CTAs (Braun and McIntosh, 1973; Green and Rachlin, 1973; Hutchison, 1973; Arwas et al., 1989; Fox et al., 1990), and because magnetic fields and rotation induce similar c-Fos patterns in the rat brainstem (Kaufman et al., 1991, 1992, 1993; Kaufman, 1996; Marshburn et al., 1997), we hypothesize that the rats may be experiencing a vestibular disturbance during magnetic field exposure comparable with the self-reports of vertigo and nausea in humans working with high-strength magnetic fields.

The inner ear may be the site of magnetic field transduction in the rat. Schenck (1992) has proposed a model in which small movements of the semicircular canals in a magnetic field would induce a magnetohydrodynamic force on the conductive endolymph, causing apparent rotation. In the absence of consistent visual or proprioceptive information, the conflicting vestibular input could cause motion sickness. We have adopted Schenck's model of vestibular disturbance as a working hypothesis for the detection mechanism in rats. This model has the virtue of relying on well established forms of sensory transduction in the vestibular system and does not require any mechanisms specific for magnetic field detection, such as biomagnetite crystals.

Although we hypothesize that the labyrinth of the inner ear is the site of magnetic field detection, other sensory pathways may be necessary or contribute to visceral stimulation mediating CTA acquisition [e.g., vagal afferents (Coil et al., 1978) or the area postrema (Ritter et al., 1980)]. The elucidation of the sensory inputs and central processing mediating the effects of magnetic 
field exposure on rats may help predict human susceptibility and responses in future generations of high magnetic field instruments such as MRI machines.

\section{References}

Arwas S, Rolnick A, Lubow RE (1989) Conditioned taste aversion in humans using motion-induced sickness as the US. Behav Res Ther 27:295-301.

Braun JJ, McIntosh H (1973) Learned taste aversions induced by rotational stimulation. Physiol Psychol 1:301-304.

Choleris E, Thomas AW, Ossenkopp K-P, Kavaliers M, Valsecchi P, Prato FS (2000) Sex differences in conditioned taste aversion and in the effects of exposure to a specific pulsed magnetic field in deer mice Peromyscus maniculatus. Physiol Behav 71:237-249.

Coil JD, Rogers RC, Garcia J, Novin D (1978) Conditioned taste aversions: vagal and circulatory mediation of the toxic unconditioned stimulus. Behav Biol 24:509-519.

Cransac H, Peyrin L, Farhat F, Cottet-Emard JM, Pequignot JM, Reber A (1997) Brain monoamines and optokinetic performances in pigmented and albino rats. Comp Biochem Physiol 116:341-349.

Fox RA, Corcoran M, Brizee KR (1990) Conditioned taste aversion and motion sickness in cats and squirrel monkeys. Can J Physiol Pharmacol 68:269-278.

Garcia J, Koelling RA (1966) Relation of cue to consequence in avoidance learning. Psychon Sci 4:123-124.

Green L, Rachlin H (1973) The effect of rotation on the learning of taste aversions. Bull Psychon Soc 1:137-192.

Hutchison SL (1973) Taste aversion in albino rats using centrifugal spin as an unconditioned stimulus. Psychol Rep 33:467-470.

Innis NK, Ossenkopp KP, Prato FS, Sestini E (1986) Behavioral effects of exposure to nuclear magnetic resonance imaging. II. Spatial memory tests. Magn Reson Imaging 4:281-284.

Kaufman GD (1996) Activation of immediate-early genes by vestibular stimulation. Ann NY Acad Sci 781:437-442.

Kaufman GD, Anderson JH, Beitz A (1991) Activation of a specific vestibuloolivary pathway by centripetal acceleration in rat. Brain Res 562:311-317.

Kaufman GD, Anderson JH, Beitz AJ (1992) Fos-defined activity in rat brainstem following centripetal acceleration. J Neurosci 12:4489-4500.

Kaufman GD, Anderson JH, Beitz AJ (1993) Otolith-brain stem connectivity: evidence for differential neural activation by vestibular hair cells based on quantification of Fos expression in unilateral labyrinthectomized rats. J Neurophysiol 70:117-127.

Kaufman GF, Shinder ME, Perachio AA (1999) Correlation of Fos expression and circling asymmetry during gerbil vestibular compensation. Brain Res 817:246-255.

Marshburn TH, Kaufman GD, Purcell IM, Perachio AA (1997) Saccule contribution to immediate early gene induction in the gerbil brainstem with posterior canal galvanic or hypergravity stimulation. Brain Res 761:51-58.

Messmer JM, Porter JH, Fatouros P, Prasad U, Weisberg M (1987) Exposure to magnetic resonance imaging does not produce taste aversion in rats. Physiol Behav 40:259-261.

Nakamura-Palacios EM, De Oliveira RW, Gomes CF (1999) Effects of diazepam or haloperidol on convulsion and behavioral responses induced by bilateral electrical stimulation in the medial prefrontal cortex. Prog Neuropsychopharmacol Biol Psychiatry 23:1369-1388.

Narasimhan PT, Jacobs RE (1996) Neuroanatomical micromagnetic resonance imaging. In: Brain mapping: the methods (Toga AW, Mazziotta JC, eds), pp 147-167. New York: Academic.

Nolte CM, Pittman DW, Kalevitch B, Henderson R, Smith JC (1998) Magnetic field conditioned taste aversion in rats. Physiol Behav 63:683-688.

Ossenkopp KP, Innis NK, Prato FS, Sestini E (1986) Behavioral effects of exposure to nuclear magnetic resonance imaging. I. Open-field behavior and passive avoidance learning in rats. Magn Reson Imaging 4:275-280.

Ossenkopp KP, Rabi YJ, Eckel LA, Hargreaves EL (1994) Reductions in body temperature and spontaneous activity in rats exposed to horizontal rotation: abolition following chemical labyrinthectomy. Physiol Behav $56: 319-324$

Parker LA (1995) Rewarding drugs produce taste avoidance, but not taste aversion. Neurosci Biobehav Rev 19:143-157.

Richter A, Ebert U, Nobrega JN, Vallbacka JJ, Fedrowitz M, Löscher W (1999) Immunohistochemical and neurochemical studies on nigral and striatal functions in the circling (ci) rat, a genetic animal model with spontaneous rotational behavior. Neuroscience 89:461-471.

Ritter S, McGlone JJ, Kelley KW (1980) Absence of lithium-induced taste aversion after area postrema lesion. Brain Res 201:501-506.

Schenck JF (1992) Health and physiological effects of human exposure to whole-body four-tesla magnetic fields during MRI. Ann NY Acad Sci 649:285-301.

Schenck JF, Dumoulin CL, Redington RW, Kressel HY, Elliot RT, McDougall IL (1992) Human exposure to 4.0-Tesla magnetic fields in a whole body scanner. Med Phys 19:1089-1098.

Shima F (1984) Circling behavior depending on striopallidal and vestibular functions. Adv Neurol 40:47-54.

Smith JC, Roll DL (1967) Trace conditioning with X-rays as an aversive stimulus. Psychon Sci 9:11-12.

Snyder D, Jahng JW, Smith JC, Houpt TA (2000) c-Fos induction in visceral and vestibular nuclei of the rat brainstem by a 9.4T magnetic field. NeuroReport 11:1681-1685.

Stephan FK, Smith JC, Fisher EM (1999) Profound conditioned taste aversion induced by oral consumption of 2-deoxy-D-glucose. Physiol Behav 68:221-226.

Weiss J, Herrick RC, Taber KH, Contant C, Plishker GA (1992) Bio-effects of high magnetic fields: a study using a simple animal model. Magn Reson Imaging 10:689-694.

Winther F, Rasmussen K, Tvete O, Halvorsen U, Haugsdal B (1999) Static magnetic field and the inner ear: a functional study of hearing and vestibular function in man after exposure. Scand Audiol 28:57-59. 